\title{
Un projet de rénovation générale
}

Le système éducatif italien

\section{Francesco De Renzo}

Traducteur : Jean-Pierre Berthollet et Anne Lazarev

\section{OpenEdition}

\section{Journals}

Édition électronique

URL : http://journals.openedition.org/ries/3173

DOI : $10.4000 /$ ries. 3173

ISSN : 2261-4265

\section{Éditeur}

Centre international d'études pédagogiques

\section{Édition imprimée}

Date de publication : 1 septembre 1997

Pagination : 83-97

ISSN : 1254-4590

\section{Référence électronique}

Francesco De Renzo, «Un projet de rénovation générale », Revue internationale d'éducation de Sèvres

[En ligne], 15 | 1997, mis en ligne le 24 juillet 2013, consulté le 03 mai 2019. URL : http:// journals.openedition.org/ries/3173 ; DOI : 10.4000/ries.3173

Ce document a été généré automatiquement le 3 mai 2019.

(c) Tous droits réservés 


\title{
Un projet de rénovation générale
}

\author{
Le système éducatif italien
}

Francesco De Renzo

Traduction : Jean-Pierre Berthollet et Anne Lazarev

1 Dans cet article, on s'est efforcé de présenter le tableau des principales questions sur lesquelles on s'interroge en Italie au sujet de l'enseignement. Le débat actuel et à venir s'appuie principalement sur le projet récent de réforme globale de l'ensemble du système éducatif, la réforme recouvrant toutes les questions fondamentales aujourd'hui en discussion. C'est pourquoi on commencera par une description générale de cette réforme; viendra ensuite une analyse plus fine des aspects particuliers que prend ici la problématique de l'éducation. Mais auparavant, il serait opportun de donner l'arrièreplan sur lequel s'établit le débat scolaire en Italie.

\section{Les raisons d'une crise}

2 Aujourd'hui plus que jamais on s'interroge sur la capacité de l'école à répondre aux nécessités d'une société complexe. Il s'agit en réalité d'une donnée commune à beaucoup de pays industriels qui sont aux prises avec les changements toujours rapides de tout le processus de production et de son organisation sociale. Chacun reconnaît désormais qu'il est nécessaire de proposer aux nouvelles générations des instruments qui permettent d'affronter ces nouvelles réalités. Le développement rapide des sociétés industrielles des dernières décennies a créé les fondements d'une production et d'une organisation sociale d'un type radicalement nouveau; la prise de conscience d'un renouvellement aussi radical est à la base de l'élaboration des fonctions futures de l'école.

En effet, telle qu'elle est, l'école ne semble pas apporter de réponses efficaces aux problèmes urgents de l'insertion dans le monde du travail, de la participation consciente aux processus innovants ou au contraire de l'exclusion. 


\section{Le cas italien}

4 Les raisons de la crise scolaire en Italie ne relèvent pas seulement des nécessités nouvelles propres aux sociétés industrialisées. Elles sont aussi liées au développement historique particulier de l'école italienne. Cette histoire a été marquée par un manque d'intérêt politique généralisé à l'égard des questions éducatives (sauf rares exceptions) et par l'incapacité qui en découle à formuler des projets, des réformes et des programmes qui puissent garantir l'efficacité et la qualité du système éducatif. On voit un peu partout des signes concrets de cette négligence : nombre réduit d'élèves diplômés, scolarité moyenne faible (six ans et deux mois), persistance de l'échec scolaire avec des taux élevés au second cycle des lycées et à l'université, faiblesse du niveau de rémunération des enseignants et abaissement de leur prestige social.

5 Cette inertie coupable s'est mêlée à l'énorme développement de la société italienne dans son ensemble. Le passage d'une société rurale à une société industrielle et postindustrielle n'a pas été accompagné par la hausse du niveau moyen de scolarité de la population, ni par la prise de conscience que les bases du développement industriel et collectif d'un pays ne peuvent être assurées que par un projet éducatif novateur et efficace. L'aménagement du système scolaire n'était pas adapté aux besoins de formation. La dernière réforme structurelle de l'école remonte aux années du fascisme. Ensuite, il n'y eut que des réformes partielles, ne modifiant rien au cursus traditionnel, en particulier au niveau des lycées.

6 Toutefois, même si certains reconnaissent dans ce pays et depuis longtemps l'importance centrale du système éducatif, l'opinion publique reste peu sensible à ce thème. À la différence de pays comme la France ou la Grande- Bretagne où le débat sur l'école reste ouvert et occupe les premières pages de la presse, les plus grands quotidiens et hebdomadaires italiens se sont très peu souciés de l'école. Les rares cas qui en font parler concernent presque toujours les comportements ou les « bonnes mœurs » : tel proviseur a renvoyé deux élèves qui s'embrassaient en classe, tel malheureux professeur a molesté quelque jeune élève et ainsi de suite. Ainsi que le notait Tullio De Mauro dans un livre paru en 1995, c'est pour des affaires de ce genre que l'école trouvait une place dans la presse et jamais pour que soient évoqués les problèmes fondamentaux, problèmes qui étaient et qui sont nombreux.

7 Disons quand même que depuis un an environ, l'école a conquis un peu d'espace tant auprès des forces politiques que dans l'attention de l'opinion publique. On doit cette attention nouvelle à l'existence d'une proposition de réforme scolaire, profonde et cohérente, présentée par le ministre actuel de l'Instruction publique, M. Luigi Berlinguer.

\section{La réforme du système éducatif}

8 Lors des campagnes électorales qui se sont récemment déroulées aux États-Unis, en Grande-Bretagne et en France, le thème de l'éducation a joué un rôle essentiel. En Italie aussi, et après de nombreuses années, le rôle du système éducatif a fait l'objet d'un vif débat lors de la campagne électorale de 1996. Mais compte tenu de la méfiance habituelle à l'égard des promesses électorales, peu nombreux étaient ceux qui imaginaient qu'on allait rapidement passer des déclarations de principe à l'élaboration d'une réforme qui réorganise l'ensemble du système éducatif italien. Or, c'est ce qui s'est passé : à peine huit 
mois après son installation au gouvernement, le ministre de l'Instruction publique a soumis à l'opinion nationale un projet radical et global, de la maternelle aux dernières années de lycée. Le projet écrit a été présenté le 14 janvier 1997. Le président du Conseil des ministres, M. Romano Prodi, le vice-président, M. Walter Veltroni, le ministre du Travail, M. Tiziano Treu et le ministre de l'Instruction publique, M. Luigi Berlinguer, prirent part à cette présentation. Leur présence témoignait de toute l'importance donnée au projet de réforme et marquait la volonté politique de la mettre en œuvre. Nouveauté aussi pour les modalités mêmes de présentation : le document de base et la loi-cadre étant le matériau proposé pour une large discussion qui permette d'arriver ensuite à un projet de loi proprement dit.

\section{Les points essentiels de la réforme}

9 En toile de fond, il y a la nécessité d'élever le niveau qualitatif et quantitatif des ressources humaines pour le développement, l'emploi et la participation démocratique à la vie de ce pays. Chacun est plus ou moins conscient du déclin progressif des résultats de l'école, largement considérée comme inadaptée et éloignée des problèmes réels et en premier lieu de ceux du monde du travail.

10 L'objectif explicite de la réforme est donc l'extension et l'amélioration de l'offre de formation de façon à garantir de bonnes bases de connaissances et la capacité à s'insérer positivement dans le processus de production et dans la société. Pour atteindre cet objectif, le projet redéfinit toute l'organisation structurelle du système éducatif.

11 Le premier point de la réforme porte sur la tranche d'âge concernée par la scolarité obligatoire. En Italie aujourd'hui, l'obligation scolaire commence à six ans, à l'école primaire, et dure huit ans, jusqu'à la fin du premier cycle secondaire. Bien que plus de $95 \%$ des familles envoient leurs enfants à l'école maternelle, celle-ci n'est pas obligatoire. La réforme au contraire rend obligatoire la dernière année d'école maternelle qui marque désormais le commencement de l'école de base (ou cycle primaire). Ce cycle se prolongera ensuite sur six années pour les élèves de 6 à 12 ans. Le niveau suivant durera trois années (élèves de 15 ans en fin de cycle) et constituera ce qu'on appelle le cycle d'orientation qui, par ailleurs, est aussi la première étape du cycle secondaire. L'école de base et le cycle d'orientation constituent le parcours de la scolarité obligatoire qui durera ainsi dix ans, au lieu de huit précédemment. Le niveau suivant est constitué par trois années de cycle supérieur, allant jusqu'à 18 ans.

12 Il ne s'agit pas seulement d'une redistribution des cycles d'études; au contraire, comme on le verra plus loin, la réorganisation des cycles s'est faite en fonction des objectifs visés par la réforme du système. 


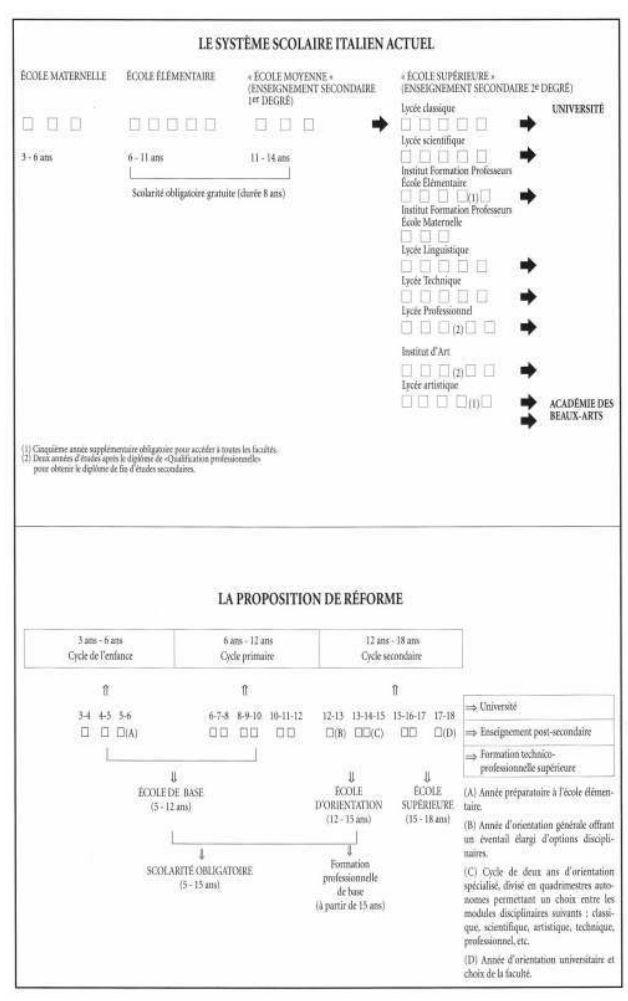

\section{L'école de base ou cycle primaire}

Le cycle primaire est réparti en trois périodes de deux ans. Il vise d'une part l'alphabétisation et d'autre part le développement de la personnalité, la formation humaine et civique. Il dure une année de plus que l'école élémentaire précédente; son programme est explicitement lié avec ce qui précède et ce qui suit. Aussi la loi-cadre affirme-t-elle : « Pendant tout le cycle primaire et au terme de chaque période de deux ans, sont introduits des séquences d'évaluation afin de prendre les mesures nécessaires à la compensation et au renforcement; l'évaluation finale prend la valeur d'un examen national ». En d'autres mots, l'évaluation sera effectuée en continu et il ne sera pas nécessaire de passer un examen comme c'est le cas aujourd'hui à la fin de l'école primaire.

\section{Le cycle secondaire}

14 C'est à ce niveau que se produisent les changements les plus importants. Il est conçu comme un seul parcours, cohérent et de six années. Actuellement, comme on le voit dans le tableau, le cycle secondaire est divisé en une " école moyenne» commune, suivie de cycles supérieurs différenciés. Le projet de réforme prévoit de diviser le cycle supérieur en trois périodes de deux ans. Ainsi disparaît l'actuelle « école moyenne » commune à tous.

Le cycle secondaire est réparti en grands domaines: humanités, sciences, techniques, technologies, arts et musique. Chaque domaine se subdivise ensuite en options. Mais, comme nous le verrons, cela ne signifie pas que l'on doive choisir son propre cursus d'études dès l'âge de douze ans. Au contraire, il y a dans l'esprit de la réforme la volonté 
de fournir les instruments de connaissance adéquats pour un choix réfléchi et pour la construction d'un parcours autonome d'apprentissage durant tout le cycle secondaire.

\section{École d'orientation}

16 C'est ainsi que sont désignées les trois premières années. Cette dénomination souligne l'objectif évoqué, à savoir fournir des instruments pour un choix réfléchi du cursus de formation et, en même temps, garantir une préparation efficace à ceux qui interrompent leurs études. La première année est identique pour toutes les options, elle se caractérise par la prédominance des enseignements fondamentaux communs à tous les domaines. On la désigne aussi sous le nom d'année d'orientation générale. La deuxième et la troisième année prévoient l'approfondissement du domaine commun et le choix de modules (ou groupes de matières) spécifiques à chaque option : humanités, sciences, techniques, etc. C'est durant ces deux années que commence le véritable parcours d'orientation, avec des contacts particuliers avec les différents domaines du savoir et du savoir-faire.

Pour ne pas pénaliser d'éventuelles erreurs de choix, on favorisera la possibilité de passer d'une option à une autre. L'apprentissage se fera à travers des modules et le passage à une option différente (par exemple, d'une option technique, informatique, à une option humaniste, classique, et vice versa) tiendra compte des modules déjà obtenus. Une importante nouveauté à cet égard consiste dans ce qu'on appelle les crédits de formation. Ces crédits, qui seront valables également durant la deuxième période de trois ans et en cas de reprise des études après une éventuelle interruption, constituent en fait la reconnaissance des études effectuées. Ils peuvent être valables dans des options différentes, en totalité ou partiellement.

18 Ces deux années aboutissent à la scolarité obligatoire et, comme l'exprime la loi-cadre, "garantissent aux élèves les connaissances, l'aptitude et l'orientation adéquates pour leurs choix de formation et de vie. Elles se terminent par un examen qui permet de poursuivre les études dans l'option choisie ».

\section{École supérieure}

19 Tout le monde s'accorde à dire que c'est le cycle ayant le plus besoin de réforme, le système dessiné par la réforme Gentile de 1924 étant demeuré tel quel. La proposition part de la nécessité de conjuguer capacités théoriques d'approfondissement et élaboration de connaissances associées à des compétences opérationnelles spécifiques et/ ou générales. En d'autres termes, la structure des diverses options - qui prévoyait, d'un côté, une instruction humaniste avec peu ou point d'espace pour la dimension concrète et, d'un autre côté, une instruction technico-professionnelle ménageant relativement peu d'espace à l'approfondissement théorique - est totalement remise en cause. S'appuyant sur d'importantes expérimentations ayant eu des effets positifs dans l'école supérieure italienne, la nouvelle école secondaire vise résolument à mieux préparer à l'insertion dans le processus de production et à l'élaboration de connaissances adaptées à la complexité de l'actuelle société de l'information. Selon le document, le projet se caractérise par un rapprochement progressif avec le monde du travail et par une incitation à une meilleure autoévaluation des capacités et affinités.

20 Ces trois dernières années devraient donc avoir une forte orientation professionnelle et culturelle. On y approfondit et y perfectionne des connaissances, des compétences et des aptitudes déjà partiellement développées durant l'école d'orientation. Ce cycle devra être 
en mesure d'orienter vers des choix fonctionnels : que ce soit vers l'université ou une formation postsecondaire non universitaire, ou vers l'insertion immédiate dans le monde du travail. Afin que ce parcours soit effectivement praticable, un vaste éventail d'options possibles est proposé, au sein des grands domaines évoqués plus haut.

En outre, l'école secondaire devra être en interaction avec les universités, les instituts de formation et les systèmes de production présents sur le territoire, où les étudiants pourront mener des séquences de formation professionnelle et des stages brefs. L'organisation de ces interactions et des collaborations liées à celles-ci devrait constituer le véritable secteur au sein duquel l'autonomie des institutions scolaires serait à même de se réaliser le plus parfaitement.

La dernière année est, elle aussi, résolument tournée vers l'orientation, outre le parachèvement des acquisitions professionnelles et culturelles.

23 La validation de l'examen final devra porter mention des études suivies, ainsi que des compétences, capacités et aptitudes acquises.

\section{La commission des Sages}

24 Un projet de ce genre suppose comme condition nécessaire de reconcevoir l'école. À cet effet, le ministère de l'Instruction publique a institué, en janvier-février de cette année, une commission technico-scientifique chargée d'identifier «les connaissances fondamentales sur lesquelles se basera l'apprentissage des jeunes dans l'école italienne des prochaines décennies». Elle est mieux connue sous le nom de "commission des Sages »: y participaient d'importantes personnalités du monde de la culture et de l'économie dont des professeurs de diverses disciplines tels Umberto Eco, Tullio De Mauro, Carlo Bernardini, le prix Nobel Rita Levi Montalcini, Clotilde Pontecorvo, le poète Mario Luzi, le cardinal Ersilio Tonini, l'acteur et réalisateur Maurizio Nichetti, mais aussi le vice-président de la Confindustria Carlo Callieri ainsi que de nombreux autres (ils étaient trente-neuf en tout).

On leur a demandé d'apporter une contribution intellectuelle, sans contrepartie financière. Les travaux de la commission sont déjà achevés et les Sages ont produit quelque cinq cents feuillets dactylographiés. Le professeur Roberto Maragliano, qui était le coordinateur, a rédigé une synthèse des questions de fond apparues dans les diverses contributions.

Dans ce rapport, l'accent est mis sur le rôle de l'école dans la formation de la personnalité individuelle et sociale de l'élève et sur ce que cela signifie en termes d'éducation à la démocratie et dans la démocratie. Évoquant plusieurs articles de la Constitution qui appellent à la participation active à la vie démocratique du pays, le texte souligne combien cette tâche est primordiale dans la perspective de l'Union européenne et en raison de relations déjà consolidées entre les diverses nations mondiales. D'où s'en suit la nécessité d'une formation non plus abstraitement théorique mais intrinsèquement liée non seulement au savoir mais au savoir-faire.

À travers la connaissance des nouvelles formes d'organisation du travail, le jeune qui sort de l'école devrait être en mesure d'accepter et d'affronter la nouvelle réalité, ce qui présuppose

« le dépassement de la "culture de l'emploi fixe" au profit d'une nouvelle vision des

changements et des évolutions professionnelles. Aussi le système éducatif devrait-il 
développer le sens des responsabilités et l'autonomie que demande le travail, les capacités éthiques et intellectuelles de collaboration avec les autres, l'esprit d'initiative permettant de résoudre des problèmes concrets et la conception de projets significatifs ».

Tout cela en collaboration avec les groupes sociaux et le monde productif.

Ces déclarations de principe esquissent une école fort soucieuse de fournir une formation qui entretienne des relations fécondes avec le monde extérieur et effectivement exploitable dans la société.

Mais si ce sont là les objectifs, il faut intervenir aussi à l'intérieur de l'organisation des savoirs dans l'école. En d'autres termes, les Sages envisagent des solutions pour identifier les savoirs fondamentaux de l'école de l'avenir et les méthodologies didactiques permettant leur transmission. En voici brièvement les caractéristiques.

Pour les méthodologies :

- abandon de la séquence leçon-étude-interrogation au profit de la construction de savoirs partagés par les enseignants et les élèves ;

- qualité des expériences théoriques et pratiques à réaliser ;

- éducation qui ne serait plus encyclopédique et comporterait la mise en œuvre de niveaux et d'objectifs diversifiés ;

- allégement important des contenus; identification des questions essentielles de chaque domaine, à connaître à fond (identification des passages incontournables et d'une série succincte de thématiques porteuses, articulations éventuelles avec d'autre niveaux dans un deuxième temps) ;

- pluralité des outils d'apprentissage : textes de bonne vulgarisation, aptes à susciter l'intérêt des élèves; activités de recherche individuelle et collective; pratiques de jeu (au-delà du cycle primaire) ; utilisation de systèmes informatiques et des technologies multimédia ;

- lien plus étroit entre l'école et la famille.

- En ce qui concerne les domaines du savoir, le document identifie les axes d'intervention suivants :

- amélioration nécessaire de l'aptitude à la production écrite et orale, afin d'acquérir une bonne compétence en matière de communication. Dans une telle perspective, il faut accorder davantage d'attention et de soin à la capacité à écrire efficacement et correctement ;

- didactique de la lecture entendue comme parcours de besoin-plaisir plutôt que comme obligation scolaire ;

- dans les disciplines scientifiques : viser au travail didactique de découverte et d'expérience directe au niveau de l'école élémentaire ;

- également dans les disciplines scientifiques, aux niveaux supérieurs, introduire une perspective critique, de nature historico-épistémologique, avec un apprentissage fondé non seulement sur les textes mais aussi sur les laboratoires et l'emploi des systèmes multimédia de simulation;

- attention particulière à l'enseignement des mathématiques, où l'on rencontre le plus d'échecs, en motivant les élèves par le plaisir de découvrir comment les mathématiques sont applicables dans diverses disciplines et dans la vie quotidienne ;

- nouvelle conception de l'enseignement de l'histoire: passer d'une vision seulement politique et où domine la succession chronologique d'événements, à la prise en compte de toutes les transformations culturelles qui concernent l'histoire des mentalités et des idées, de la littérature, de l'art, de la musique. Introduction d'une pluralité d'instruments: 
banques de données, images, sources orales, matériel cinématographique et audiovisuel, reconstructions virtuelles, jeux de rôle, etc. ;

- espace adéquat donné aux sciences sociales, à travers des «blocs thématiques » en rapport direct avec la vie sociale actuelle (par exemple: PIB et déficit, systèmes politiques et électoraux, etc.) ;

- valorisation de l'instruction civique davantage prise en compte dans l'évaluation scolaire, introduction d'éléments d'esprit critique, incitation aux débats et aux discussions ;

- garantir le rapport avec la tradition classique, de manière à faire apprécier aux jeunes l'héritage culturel dans lequel ils vivent : patrimoine commun que l'on peut s'approprier par d'autres moyens que l'apprentissage $d u$ grec ou du latin, réservés comme options particulières ;

- nécessité d'étudier le $\mathrm{XX}^{\mathrm{e}}$ siècle (réforme déjà mise en œuvre par un décret ministériel) ;

- étendre la philosophie à tous les établissements et pour les publics âgés de plus de 15-16 ans en tant qu'ensemble d'idées porteuses pour la constitution de l'identité des jeunes et pour leur réflexion sur le rapport au monde. Durant les années suivantes, elle serait enseignée par thèmes tels que l'éthique, la logique, etc. L'enseignement actuel, comme forme de reconstruction historique, n'a pas lieu d'être hors des lycées ;

- anglais fondamental et non littéraire, en tant que langue de communication, à proposer dès le cycle primaire. Durant les années suivantes, adjoindre l'étude efficace et liée à la culture d'une ou plusieurs langues de la communauté européenne ;

- extension et insertion dans le parcours éducatif des arts musicaux et visuels (cinéma, etc.), pour développer l'aptitude à apprécier, élaborer et produire des expériences culturelles nécessaires à une formation plus vaste et à une connaissance plus large de la réalité.

\section{Les réactions au projet de réforme}

Les réactions au projet de réforme ont été fondamentalement positives. La nécessité d'un changement était perçue par tous et les finalités de la réforme semblent répondre à des questions concrètes. On a relevé notamment le caractère positif de l'attention portée à une éducation étroitement liée au monde productif et à une formation permettant de faire des choix réfléchis et d'élaborer des cursus d'études et des parcours de vie autonomes. Le parcours d'orientation vers le travail et vers l'université ainsi que les contenus hautement qualifiants en matière de formation et de professionnalisation ont été jugés adaptés aux exigences de croissance productive et sociale du pays.

Le début de la scolarité obligatoire à cinq ans, son extension jusqu'à quinze ans et le caractère unifié de l'enseignement de base sont des points sur lesquels le consensus a été à peu près unanime.

Les critiques n'ont pas manqué toutefois. Les plus importantes ont concerné l'absence d'indication sur la provenance des ressources financières nécessaires à la mise en œuvre du projet.

À signaler ensuite, une mauvaise évaluation des difficultés concernant la formation nécessaire des enseignants pour affronter une révolution aussi radicale de l'organisation scolaire et de l'école. Les enseignants ont été quelque peu désorientés et, au début, incapables d'évaluer la réforme avec sérénité. Nombreux sont ceux - surtout parmi les professeurs des écoles «moyennes» et «supérieures»- qui ont exprimé leur préoccupation et leur perplexité sur leur place dans les futures structures scolaires. Par ailleurs, le corps enseignant soulignait la nécessité d'une formation spécifique et 
approfondie pour répondre à l'objectif de qualité visé, c'est-à-dire aussi un engagement de ressources humaines et de fonds significatifs - point particulièrement important dans un pays où les professeurs sont parmi les moins payés au monde et où les dépenses pour l'instruction sont parmi les plus faibles. C'est là un des points critiques de la réforme, sinon le plus important. Motiver le corps enseignant, lui donner les moyens de travailler efficacement et lui offrir les gratifications, y compris économiques, qu'on lui a souvent refusées dans le passé : telles sont les difficultés réelles de la mise en œuvre de la réforme. Pour la petite histoire, il faut signaler les vives critiques que les forces politiques de l'opposition ont adressées au ministre. Plus que le projet lui-même, ces dissensions concernaient surtout la reconnaissance du rôle des écoles privées et de leur financement. Elles se mêlaient à des critiques sur la gestion de la politique éducative du ministre, accusé de dirigisme.

\section{La formation des adultes et la formation permanente}

Parmi les divers aspects du retard italien en matière d'instruction, le faible niveau d'instruction générale est le plus significatif. Pour des raisons qui remontent très loin dans le temps, on est parvenu à l'école obligatoire selon un processus très lent.

De fait, le cadre socioculturel fourni par les statistiques sur l'éducation est celui d'un pays où les jeunes générations sont en moyenne trois à quatre fois plus instruites que les générations précédentes et où presque la moitié de la population de plus de quatorze ans n'a même pas le diplôme délivré à la fin de la scolarité obligatoire. Les effets de cette faible alphabétisation se manifestent non seulement dans le nombre restreint de lecteurs de livres (seuls $35 \%$ des Italiens déclarent lire au moins un livre par an) ou de journaux, mais aussi dans la carrière scolaire des enfants de parents peu alphabétisés. Des enquêtes et des recherches internationales ont en effet démontré que le niveau d'étude des parents est en rapport direct avec les résultats scolaires des enfants. Cette influence, déjà évidente à l'école élémentaire et "moyenne", devient encore plus perceptible aux échelons supérieurs. Les trois-quarts de ceux qui parviennent à la fin de leurs études universitaires ou scolaires sont les enfants des parents, peu nombreux, qui ont fait de même (en tout environ $22 \%$ ). Les autres, la très grande majorité, s'arrêtent avant.

À ce tableau, il faut ajouter la diminution progressive d'une main d'œuvre spécialisée en faveur d'une main d'œuvre à plus bas coût, ou d'une main d'œuvre aux capacités professionnelles plus élaborées. Ce sont là aussi de vieilles questions en Italie. Des chercheurs comme Tullio De Mauro ne manquent pas de rappeler, depuis trente ans déjà, que la formation des adultes est un devoir social, civique, politique et même économique pour la république italienne. À l'heure actuelle, dans la loi-cadre de réforme de l'école, il est fait explicitement référence à cette formation des adultes, impliquant officiellement le ministère du Travail: une formation qui voudrait permettre à des millions d'adultes d'obtenir le diplôme de fin de scolarité obligatoire ou celui de fin d'études secondaires, mais qui se voudrait aussi une «formation permanente », étant donné les besoins de mise à jour et de formation continue. L'article 13 (formation des adultes), deuxième et troisième alinéas, de la loi-cadre dit ceci :

«2. Les institutions scolaires, sur la base notamment de demandes ou d'accords avec les organismes locaux, mettent en place, seules ou en association, des programmes de formation spécifiques, en particulier pour l'obtention du diplôme 
de fin d'école obligatoire ou de celui de fin d'études secondaires destinées aux adultes.

«3. Les établissements du secondaire peuvent instituer des cours de formation et d'approfondissement pour les personnes exerçant des professions pour lesquelles le diplôme universitaire de Laurea ${ }^{1}$ n'est pas requis. Ces cours sont organisés selon des modalités compatibles avec les activités professionnelles des participants ».

40

régulière des adultes déjà inscrits dans le monde du travail et ayant derrière eux une expérience négative de leur rapport à l'école, constitue une difficulté supplémentaire. Là encore, une formation spécifique des enseignants est indispensable. En effet, enseigner aux adultes suppose l'élaboration de stratégies et d'instruments didactiques ciblés.

41 C'est une donnée positive que, dans le schéma général du nouveau système éducatif, la formation des adultes ait obtenu l'attention requise. Les modalités de mise en œuvre seront, encore une fois, décisives pour la réussite de cette partie du projet.

\section{La déperdition scolaire}

Le problème de la déperdition scolaire est directement lié à la formation des adultes. Ce n'est pas un problème résolu. En Italie, dix enfants sur cent n'arrivent pas à la fin de la scolarité obligatoire. À l'école "supérieure", le moment des plus grands échecs et abandons se situe lors de la première année et plus spécifiquement dans les établissements technico-professionnels, avec des pointes atteignant les $30 \%$. Mais cela continue les années suivantes. Seuls $49 \%$ des jeunes obtiennent un diplôme de fin d'études secondaires: une des moyennes les plus basses de l'Union européenne et des pays de l'OCDE.

Les données relatives à l'hémorragie universitaire sont encore pires : seuls trois étudiants sur dix inscrits en première année arrivent à la fin de leurs études, les sept autres abandonnent presque aussitôt. Comme nous l'avons déjà vu, toute l'architecture du projet, avec l'accent fortement mis sur le rôle d'orientation de l'école, tend précisément à éviter de telles issues négatives. Le caractère unifié de l'école de base, l'école d'orientation et la dernière année de l'école supérieure visent expressément à éviter les échecs dus à des erreurs de choix. Le rappel constant du lien indispensable de l'école avec l'université et avec la réalité économique du territoire devrait réduire au minimum les risques de choix erronés dus à une formation et une information défaillantes.

Par ailleurs, dans le cadre de l'éducation permanente, le projet de réforme prévoit aussi un droit à la formation pour ceux qui décident de s'arrêter une fois l'école obligatoire terminée. L'article 11 affirme que ce droit est reconnu jusqu'à dix-huit ans pour l'obtention d'une qualification, au moyen d'une offre accrue en formation professionnelle extrascolaire et de l'interaction entre cette offre et les programmes d'enseignement.

\section{Les rapports système éducatif/monde du travail}

Selon un récent sondage, les critiques les plus vives concernent la relation entre l'école et le monde du travail. Comme on l'a vu, la réforme fait des pas significatifs à cet égard. Le projet de réforme tout entier s'appuie essentiellement sur un rapport renouvelé et efficace entre le système éducatif et le marché du travail. Outre la possibilité d'expériences directes menées entre l'école et le monde de la production, la réforme 
tente de dessiner aussi un parcours de sortie du système éducatif. L'une des orientations, déjà évoquée, consiste à renforcer la formation professionnelle. En plus des régions, celleci devra être gérée par les établissements secondaires et concernera aussi les personnes ayant obtenu le diplôme de fin d'études secondaires. Bien qu'il ne se situe pas dans une continuité directe, un lien avec les universités est prévu en ce sens que, là aussi, on devrait pouvoir avoir accès à des crédits de formation pris en compte pour une poursuite éventuelle des études.

L'autre orientation est la relation avec l'université. Là aussi les rapports devront être constants et commencer dans les dernières années de l'enseignement secondaire. D'une part, de tels contacts devraient permettre de mieux évaluer les aptitudes et la vocation des élèves, pour éviter les nombreux échecs universitaires, largement dus à un manque d'information et d'orientation. D'autre part, le rôle de l'université devrait apparaître dans la formation du personnel enseignant, comme cela est d'ailleurs prévu depuis longtemps déjà dans diverses lois.

Toutefois, la prise de conscience du fait que le mythe de l'emploi « fixe», du travail qui dure toute la vie, est révolu, constitue un élément de nouveauté supplémentaire. À cet égard, la formation devra préparer les étudiants à l'idée qu'ils pourront éventuellement changer de profession plusieurs fois au cours de leur vie. Cet axiome est clairement souligné dans l'introduction de la réforme.

Les connaissances et les compétences spécifiques risquent de vieillir rapidement, si elles ne sont pas soutenues d'une manière opportune par des capacités autonomes de formation et de mise à jour. Aussi, non seulement le système doit-il préparer à une spécificité professionnelle, mais il doit aussi se caractériser par son aptitude à fournir une formation culturelle favorisant la maturité de la personne. Comme le disent entre autres l'économiste Nicola Rossi et le sociologue Renato Mannheimer, cela semble en définitive la formation la plus adéquate pour affronter le marché du travail actuel qui exige souplesse et capacité d'adaptation plutôt qu'une préparation spécifique.

\section{La parité école publique/ école privée}

Un autre élément de débat, fortement ressenti et largement discuté, notamment dans la presse, concerne la perspective de parité entre l'école publique et l'école privée.

La demande d'une parité de traitement part du constat que les écoles privées, fournissant un service public, doivent avoir accès aux financements de l'État. Cela peut apparaître comme un sujet marginal, étant donné qu'en Italie l'école privée (en majorité catholique) est largement minoritaire et ne couvre même pas $10 \%$ de l'offre de formation. Cependant, il existe de fortes poussées Politiques dans cette direction. C'est à cet effet qu'a été constituée une commission ayant pour tâche de dessiner les axes de la mise en œuvre pratique de ce projet. À l'heure où nous écrivons, le ministère a proposé un projet de loi qui est actuellement soumis aux forces politiques et sociales. Ce projet de loi prévoit la possibilité que l'État accorde des fonds aux écoles privées à un niveau non inférieur à $35 \%$ du coût moyen des dépenses assumées par l'État pour les élèves des écoles publiques. En outre, les parents peuvent soustraire de leurs impôts jusqu'à un million de lires par enfant sur les dépenses faites pour les livres et les matériels didactiques. La proposition soumet cependant l'attribution de ces fonds au respect des normes éducatives générales, 
à des règles de recrutement des enseignants plus claires et à l'accueil sans discrimination de tout élève, y compris les élèves handicapés.

51 Les oppositions sont nombreuses et très fortes. Un récent sondage publié par le Corriere délia Sera du 13 juillet 1997 révèle que 15,4\% seulement des interviewés sont favorables à ce que l'État finance les écoles privées, qu'elles soient laïques ou religieuses. Mais des résistances encore plus fortes viennent d'intellectuels, de juristes et de diverses forces politiques. Au-delà des motivations d'ordre idéologique et/ou politique, qui ont pourtant leur poids, on rappelle que l'alinéa 3 de l'article 33 de la Constitution déclare que « les organismes et les personnes privées ont le droit de créer des écoles et des instituts de formation, sans charge pour l'État ». Si l'on ne modifie pas l'article de la Constitution, il semble impossible que l'État puisse financer des écoles privées.

Le débat, extrêmement vif, bat son plein, les forces politiques prenant des positions qui ne coïncident pas avec la dualité gouvernement/opposition. Bref, la question est encore ouverte, même si le ministre semble avoir l'intention de promulguer la loi sur la parité. En attendant, au niveau local, certaines administrations prennent déjà ce chemin. C'est ce qui s'est produit, par exemple, à Rome où la municipalité a débloqué plus de 15 milliards de lires en faveur des écoles privées de la ville.

\section{Le système national d'évaluation}

53 La nécessité d'avoir des paramètres et des critères unifiés pour évaluer l'efficacité scolaire se traduit par la préparation de la mise en place d'un service national chargé d'évaluer la qualité de l'enseignement. Dans ce cas aussi, on a constitué une commission dont font partie d'éminents spécialistes et pédagogues. Le président de la commission est le professeur Aldo Visalberghi, qui fut directeur du CEDE (Centre Européen de l'Éducation). La commission a pour tâche de déterminer les critères permettant d'évaluer l'efficacité du système éducatif dans tous ses aspects.

Les principaux axes d'intervention sont:

- l'identification des paramètres significatifs, en relation avec les principaux rapports internationaux sur l'instruction ;

- la mise au point d'instruments permettant de définir les compétences et les aptitudes acquises par les élèves ;

- des systèmes statistiques automatisés visant l'évaluation, comportant des banques de données et des fichiers facilement accessibles;

- la collaboration avec des organismes d'évaluation périphériques déjà existants ;

- la mise en place d'une structure de soutien pour les épreuves d'examen ou les vérifications concernant un nombre important d'élèves ;

- la collaboration avec les institutions internationales analogues ;

- la définition de procédures standards pour le traitement des données d'évaluation;

- la détermination de critères et de modalités générales pour l'évaluation du personnel de direction et du corps enseignant ;

- la formation d'experts sur les problèmes de l'évaluation.

Comme on le voit, les tâches sont nombreuses et, dans une intervention publique auprès du quotidien La Repubblica, le président de la commission s'inquiétait déjà de ce que le manque de ressources puisse compromettre la réussite du projet. 

aptitudes acquises par chaque élève), pour le système de crédits de formation, de même que pour l'évaluation générale de la qualité de formation des différents établissements publics et privés, le service national d'évaluation de la qualité de l'enseignement revêt lui aussi une importance capitale dans le nouveau système éducatif. délais de réalisation devraient être rapides. Dès l'année scolaire 1998-99, nous devrions accueillir les premiers élèves de la nouvelle école.

Une donnée est toutefois certaine : on parle beaucoup plus d'éducation aujourd'hui que par le passé et on peut consulter directement sur Internet ${ }^{2}$ les documents officiels du débat.

\section{NOTES}

1. Équivalent d'un diplôme baccalauréat $+4,5$ ou 6 .

2. Adresse du site : http :/www.bdp.si.it/

\section{RÉSUMÉS}

En Italie, les responsables et l'opinion publique manifestent un intérêt nouveau pour la qualité du système éducatif. Le projet de réforme scolaire actuellement en débat illustre cette évolution tant par son ampleur que par l'exigence, constamment soulignée, d'une adaptation de l'école aux transformations de la société.

\section{INDEX}

Mots-clés : politique éducative, réforme de l'enseignement, système éducatif Index géographique : Italie

\section{AUTEURS}

\section{FRANCESCO DE RENZO}

Observatoire linguistique et culturel italien, département d'études linguistiques et littéraires, Université La Sapienza, Rome, Italie. 